\title{
Pyrrole hemithioindigo antimitotics with near-quantitative bidirectional photoswitching photocontrol cellular microtubule dynamics with single-cell precision
}

\author{
Alexander Sailer ${ }^{\dagger}$,Joyce C.M. Meiring ${ }^{\S}$, Constanze Heise ${ }^{\dagger}$, Linda N. Pettersson ${ }^{\dagger}$, Anna Akhmanova $^{\S}$, \\ Julia Thorn-Seshold ${ }^{\dagger}$, Oliver Thorn-Seshold ${ }^{*,+}$ \\ ${ }^{\dagger}$ Department of Pharmacy, Ludwig-Maximilians University of Munich, Munich 81377, Germany \\ ${ }^{s}$ Cell Biology, Department of Biology, Faculty of Science, Utrecht University, Utrecht 3584, The Netherlands \\ photopharmacology • cytoskeleton • microtubule dynamics • photoswitch • photochromism • cytotoxicity • hemithioindigo
}

\begin{abstract}
Photoswitchably bioactive reagents, known as "photopharmaceuticals", promise powerful applications in high-precision biological research. Yet most photoswitch scaffolds cannot be quantitatively bidirectionally photoisomerised, so they suffer from residual background activity that can confound experiments. We rationally designed photopharmaceuticals using the emerging near-quantitative photoswitch pyrrole hemithioindigo (PHTubs), to isomer-specifically inhibit the cytoskeletal protein tubulin. These PHTub reagents allow simultaneous visible-light imaging and photoswitching in live cells, where they could be used for cell-precise photomodulation of microtubule dynamics, and photocontrol over cell cycle progression and cell death. This is, as far as we know, the first use of a hemithioindigo photopharmaceutical for high-spatiotemporal-resolution biological control in live cells. This work opens up new horizons for high-precision microtubule research using PHTubs; and shows the cellular applicability of the near-quantitative photoswitch pyrrole hemithioindigo as a valuable scaffold for photocontrol of a range of other biological targets.
\end{abstract}

\section{INTRODUCTION}

Optically targeted approaches to manipulate biological systems have revolutionised diverse research fields, by enabling the non-invasive patterning of protein activity with resolution on the spatial and temporal scales inherent to cellular functions. The high precision studies that optical approaches allow have particular promise for systems whose spatiotemporal regulation is key to their functions: such as action potential firing in neuroscience, or cytoskeleton structure and dynamics in cell biology. ${ }^{1-3}$ The major optical approaches are optogenetics, photouncaging, and photopharmacology. Photopharmacology offers both distinct performance and a unique application space. Unlike photouncaging, photopharmaceuticals can be modulated reversibly, which is vital for avoiding a build-up of background bioactivity that could compromise biological readout; they feature near-instantaneous photoresponse without generating toxic uncaging byproducts; and their photoresponse wavelengths are typically more biologically-compatible as well as more tunable than those of caging groups. ${ }^{4,5}$ Unlike optogenetics, small molecule photopharmaceuticals are easily transitioned between different biological systems as they do not require genetic engineering (thereby also having potential for therapeutic applications); and crucially, they can target proteins which do not permit functional photocontrol by fusion constructs, such as the cytoskeletal scaffold proteins tubulin and actin. ${ }^{3,5,6}$ Since the spatiotemporally-precise orchestration of cytoskeleton structure and dynamics is critically important in hundreds of biological processes, the development of cytoskeleton-modulating photopharmaceuticals has emerged as a valuable goal of research in recent years. ${ }^{7,8}$ These hold promise for basic research into anisotropic processes including intracellular transport, mechanostasis and cell motility, as well as for applied research in anti-invasive and anti-proliferative strategies for cancer pharmacology. ${ }^{7,9,10}$

The development of photopharmaceuticals has however been held back by the limited range of photoswitches that permit bioactivity photoswitching in cells, and by the drawbacks of the known scaffolds. An "ideal" photoswitch would permit efficient, nearcomplete, bidirectional photoisomerisations in situ in live cells under practical conditions, to allow efficient and precise photopatterning of its isomers. An ideal photopharmaceutical would further need to decorate this scaffold to create a ligand with near-zero bioactivity as one photoisomer and potent bioactivity as the other photoisomer. The vast majority of cellularly-applicable photopharmaceuticals rely on azobenzenes, with diarylethenes, fulgides and hemithioindigos playing minor roles (Fig 1a), yet these scaffolds are far from the ideal. In this work we expand the range of cellular photoswitch scaffolds, solving two performance problems that azobenzenes in particular fail to address. In doing so we provide both a useful new reagent for photocontrol of the microtubule (MT) cytoskeleton, and a general advance for practical photopharmacology.

The first major disadvantage of current photoswitches that we focus on, is that in general, they cannot be quantitatively bidirectionally photoisomerised in biological settings, which reduces 
their power to photopattern biological activity. Taking azobenzene as the most important example, its largest typical change of photostationary state (PSS) composition is around 4-fold (ca. 20:80 trans:cis under $370 \mathrm{~nm}$ and ca. 80:20 trans:cis under $450 \mathrm{~nm}$, though new polysubstituted or heteroaromatic azobenzenes perform better). ${ }^{1,11-13}$ Therefore, even if only one of an azobenzene's two isomers is at all biologically active, photoswitching in the cellcompatible wavelength range $(>360 \mathrm{~nm})$ can, in a best-case scenario, achieve just 4-fold photocontrol over the concentration patterning of the bioactive species. We define this useful quantity of the photoswitch scaffold as its maximum functional dynamic range or FDR $_{\max }$. Note however, that typical photopharmaceuticals have substantial residual bioactivity in the less active isomer, and so their practical FDR cannot approach the photoswitchability of bioactivity limit that this $\mathrm{FDR}_{\max }$ represents. The second disadvantage we focus on is that the fixed-wavelength lasers available to biologists cannot deliver even these "best-case" isomerisations, since typical confocal microscopes feature a subset of the 405, 442, 488, 514, 561, and $647 \mathrm{~nm}$ laser lines.

The combination of these two disadvantages can easily ruin the performance of biologically well-designed photopharmaceuticals. As an example, the azobenzene photopharmaceutical PST-1, featuring an exceptional $>10,000$-fold difference of binding potency between the pure photoisomers ${ }^{14}$, gave a FDR $\max$ of just 9-fold with "best-case" photoisomerisations $(380 / 525 \mathrm{~nm})$ which is even comparatively high for azobenzenes; but when using biologically available laser lines, the best FDR that can be reached is just 3-fold (405/514 nm): a drastic loss of potential for an otherwise excellent photopharmaceutical. $^{3}$

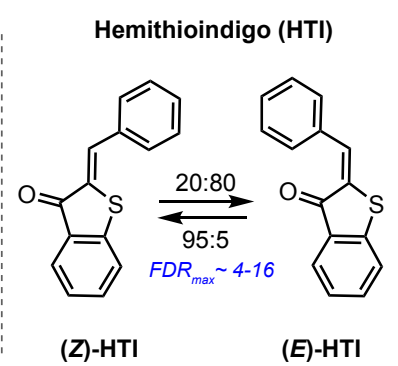

(Z)-SBT

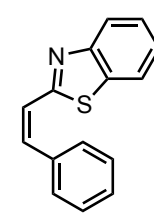

(Z)-HTI

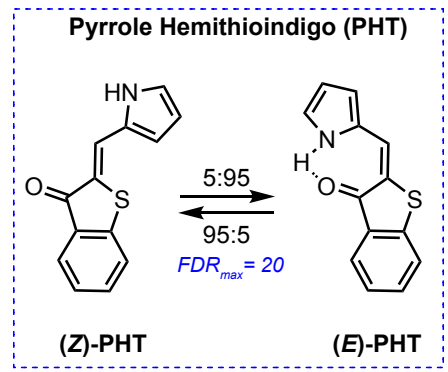<smiles>COc1cc2c(c(OC)c1OC)-c1c(ccc(OC)c1OC)[C@@H](N)CC2</smiles>

(S)-colchicine

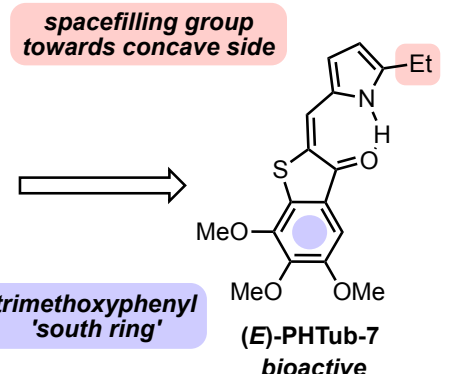

d

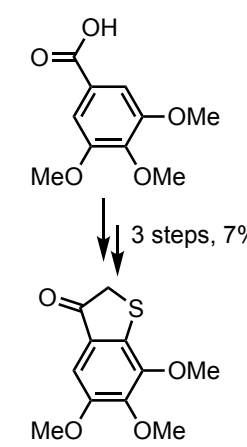

C<smiles>CCc1ccc(/C=C2\Sc3cc(OC)c(OC)cc3C2=O)[nH]1</smiles><smiles>CCc1cc[nH]c1/C=C1\Sc2cc(OC)c(OC)c(OC)c2C1=O</smiles>
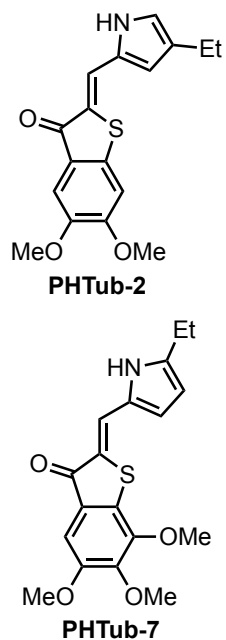
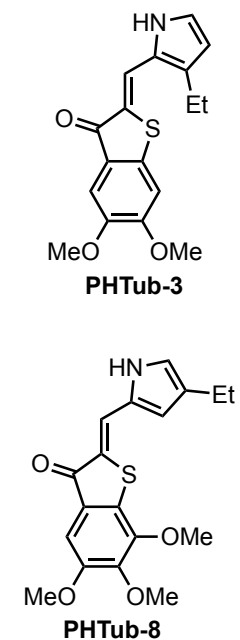

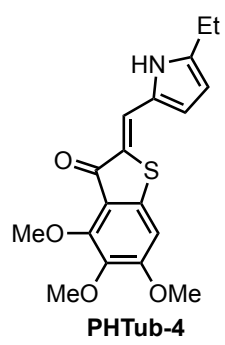<smiles>CCc1c[nH]c(/C=c2/sc3cc(OC)c(OC)c(c3OC)c2=O)c1</smiles>

Figure 1 - Design and synthesis of PHTubs: (a) Photoswitchable scaffolds azobenzene, styrylbenzothiazole, hemithioindigo, and pyrrole hemithioindigo, with typical best photostationary state isomer ratios achieved under photoswitching in the biologically compatible wavelength range in physio-

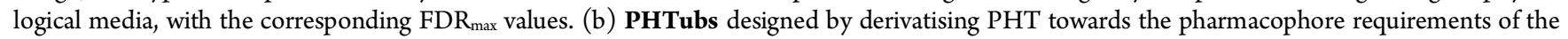
colchicine binding site on tubulin. (c) Structures of PHTubs synthesised and tested in this study. (d) Synthesis of representative reagent, PHTub-7.

To create practically useful photopharmaceuticals for the MT cytoskeleton, we therefore focussed on photoswitch scaffolds with high FDR max $_{\text {ax }}$ that also match to the biologically accessible visible- light lasers. Previous explorations of $\mathrm{C}=\mathrm{C}$-based photoswitches (405 nm-switchable styrylbenzothiazole ${ }^{15}$ as well as visible-lightswitchable hemithioindigos ${ }^{16}$; Fig 1a) have highlighted their 
robustness to glutathione (GSH) as well as their tolerance of tautomerisable polar functional groups (e.g. para-hydroxy groups) that are important for ligand-target interactions. These features make $\mathrm{C}=\mathrm{C}$-based photoswitches attractive for further research, particularly compared to azobenzenes; which are known to suffer GSH lability, and where tautomerisable functional groups counteract their photoisomer patterning. ${ }^{17,18}$

Newhouse and Zweig recently developed C=C-based pyrrole hemithioindigos (PHT, Fig 1a), which have FDR max $_{\max }$ of ca. 40 in organic solvents due to enhanced band separation between $E$ and $\mathrm{Z}$ isomers associated to an intramolecular pyrrole $\mathrm{NH}$... $\mathrm{O}=\mathrm{C}$ carbonyl hydrogen bond in the metastable $E$ isomer. ${ }^{19,20}$ While the photostability and resistance to GSH of this photoswitch were encouraging signs for biological use, their report described insolubility and poor/zero photoswitching performance in increasingly aqueous media. ${ }^{19}$ However, we previously observed that polymethoxylated derivatives of otherwise low-solubility scaffolds can be photoswitched repeatedly in situ in cells, giving unexpectedly high photopatterning performance which mirrors that seen in apolar media. ${ }^{21}$ We attributed this to cellular sequestration of the hydrophobic photoswitches primarily in water-excluded environments: allowing photoswitching in lipid environments, and comparatively slow cell-average rates of spontaneous $E$-to- $Z$ relaxation, yet with rapid exchange supplying the photopatterned isomer ratio to the cytosol. ${ }^{21}$ We reasoned therefore that a sufficiently soluble pyrrole hemithioindigo photopharmaceutical might be able to apply a cellular FDR approaching this high $\mathrm{FDR}_{\max }$, as its photostationary state equilibria (PSSs) would reflect those determined in apolar media.

This we determined to test experimentally by designing PHTubs: PHT-based photopharmaceuticals targeting the colchicine binding site on tubulin, for cellular use as photoswitchable antimitotics and cytoskeleton inhibitors: aiming at high-FDR and high-spatiotemporal-resolution photocontrol of cell cycle, cell viability, and cellular MT dynamics.

\section{RESULTS}

\section{Design and Synthesis}

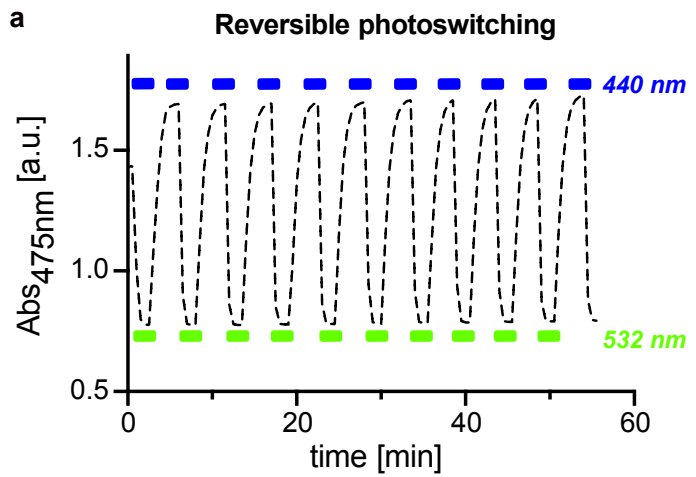

We designed PHTubs as closely isosteric PHT-based analogues of the MT inhibitor colchicine. The colchicine pharmacophore consists of two aryl blades, with key characteristics being (i) a "southern" di- or tri-methoxyaryl ring, fixed close to (ii) a "northern" ring projecting a methoxy or ethyl spacefilling group (Fig 1b). ${ }^{22}$ We chose the thioindoxyl half of the PHTubs as the southern ring to permit triple substitution, leaving the pyrrole ring to bear the single ethyl group. We created nine PHTubs to scan which orientations of the di/trimethoxy south substituents and the ethyl north substituent would deliver both the strongest potency and the greatest isomer-dependency of bioactivity. Of these, we expected that the 3'-ethyl substituted compounds (PHTub-3/6/9) would not bind potently in either isomer due to the mismatched orientation of the ethyl group (Fig $\mathbf{1 b}$ ). We therefore expected PHTub-3/6/9 would serve as useful "permutation controls" (designed-inactive compounds). We routinely use permutation controls to test for nonspecific bioactive effects: both those known for pan-assay interference compounds (PAINS) ${ }^{23}$ which should also be anticipated for hydrophobic photoswitches (e.g., promiscuous binding, aggregation on proteins, or membrane disruption), as well as nonspecific scaffold toxicity or phototoxicity under illumination. ${ }^{16}$ If permutation controls are inactive, this supports that bioactivity seen for designed-active PHTubs is target-specific and isomer-specific, and does not involve these nonspecific effects. Furthermore, we also synthesised an $\mathrm{N}$-methylated control PHTub-NMe; we likewise expected this to be inactive due to the clash of the N-methyl group with the binding pocket wall, ${ }^{24}$ but were interested to study the effects of suppressing the $\mathrm{NH} \ldots \mathrm{O}=\mathrm{C}$ hydrogen bond upon its photochemistry and bioactivity (Fig 1c).

PHTub-1-9 and N-methylated control PHTub-NMe were synthesised in good yields in four to eight steps. In brief, reduction of acetylpyrroles followed by Vilsmaier-Hack formylation gave ethylpyrrole carboxaldehydes. Thioindoxyls were accessed by intramolecular Friedel-Crafts acylation of the 2-(phenylthio)acetic acid, or by base treatment of 2-(methylthio)benzamides. Piperidine-catalysed aldol condensation of the thioindoxyls with the pyrrole carboxaldehydes gave the target PHTubs (Fig 1d and Supporting Information).

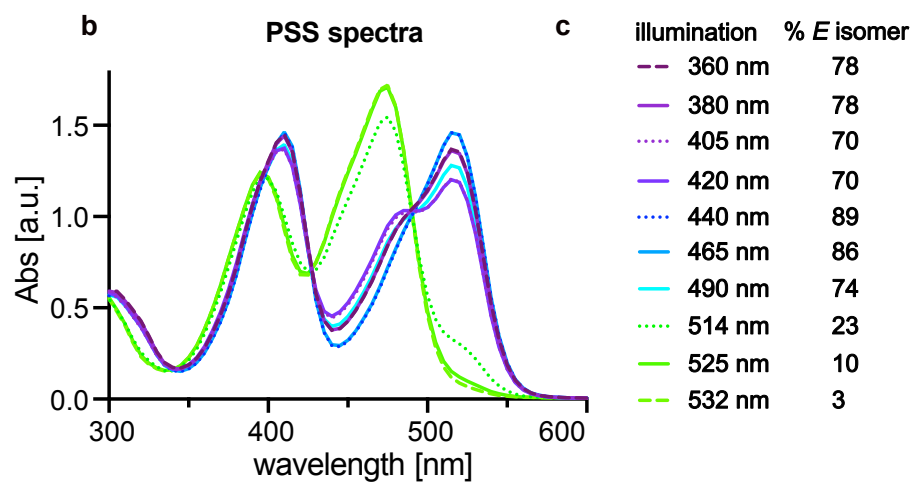

Figure 2 - Photoswitching of PHTubs: (a) PHTubs can be robustly photoswitched from a majority-Z to a majority-E PSS using $440 \mathrm{~nm}$, and cycled between this PSS and the majority-Z PSS at $532 \mathrm{~nm}$. (b-c) PSS absorbance spectra and isomeric compositions of PHTub-7 when illuminated in the biologically-compatible wavelength range.

\section{Photoswitching}

The PHTubs could be photoswitched in dry or in $25 \%$ aqueous polar media, under air, over multiple cycles without degradation (Fig 2a, Fig S2-S3). Anticipating that they would probably concentrate in water-excluded cellular environments due to their lipophilicity, we therefore expected that PHTubs could display good cellular photoswitchability at $430-450 \mathrm{~nm}$ (mostly-E) vs $510-530 \mathrm{~nm}$ (mostly- $Z$ ). By avoiding photoresponse to $>550 \mathrm{~nm}$ 
we also expected that they could permit orthogonal imaging in the red fluorescent protein (RFP) channel at $561 \mathrm{~nm}$ (Fig 2b-c).

Matching expectations from Newhouse and Zweig ${ }^{19}$, the band separation of PHT isomers and the $\mathrm{FDR}_{\max }$ were dependent on the solvent's capacity to interrupt the internal H-bond (Table S1, Table S3), decreasing from ca. $45 \mathrm{~nm}$ separation in dry $\mathrm{MeCN}$ $\left(\mathrm{FDR}_{\max }\right.$ ca. 20 -fold at $440 / 525 \mathrm{~nm}$ for all compounds) to ca. $30 \mathrm{~nm}$ in 75:25 DMSO:PBS (FDR max $_{\max }$ compound-dependent and below 10-fold). The $N$-methylated control PHTub-NMe displayed photoswitching similar to a phenyl hemithioindigo in both dry organic solvents and DMSO:PBS mixtures, highlighting the role of the $\mathrm{NH} \ldots \mathrm{O}=\mathrm{C}$ hydrogen bond in reaching a high FDR in apolar media (note that Newhouse and Zweig's $N$-methylated PHTs were reported not to display photoswitching $\left.{ }^{19}\right)$. Unlike the 3-/4-ethylated compounds which had all- $Z$ dark-adapted states, the 5-ethylated PHTub-3/6/9 seemed to have substantial $E$ isomer proportion in the dark-adapted state (see Supporting Information). Typical $E$-to- $Z$ relaxation halflives were in the hours to minutes range in most media with a clear trend of decreasing with solvents' polarity and ability to disrupt hydrogen bonds (Fig S4). They resisted challenge by $10 \mathrm{mM} \mathrm{GSH}$ over hours in the stable Zisomer state, and the $E$ isomer was also found to be unresponsive to GSH challenge over repeated cycles of $Z-E$ isomerization and thermal relaxation (Fig S6). This contrasts favourably to the GSH-mediated destruction of similarly-substituted Z-azobenzenes. $^{24}$

We therefore began exploring the applicability of PHT as an in situ-photoswitchable scaffold for cell biology, through the use of PHTubs as photoswitchable MT inhibitors.

\section{Structure-and-photoisomer-activity relationship}

Prolonged microtubule inhibition blocks cell proliferation and ultimately results in cell death. ${ }^{25}$ Therefore, we initially assessed the cellular bioactivity of the PHTubs by assaying their antiproliferative activity, in 44 hour assays in both HeLa human cervical cancer and Jurkat T-cell leukaemia cell lines, under ongoing pulsed wavelength-specific illuminations designed to test both $E$ and $Z$ PHT isomers (Table 1). ${ }^{3}$

PHTub-4-6 gave essentially only low-potency effects that match expectations for weak and nonspecific cellular toxicity of aggregation-prone compounds near their solubility limit. We concluded that they could not bind to tubulin, due to a steric clash of their south ring's outer methoxy group with the T7 loop of the colchicine site. ${ }^{14,16}$ Yet, since both di- and tri-methoxyaryl rings should be tolerated in the southern binding pocket, ${ }^{22}$ we expected that deleting this methoxy group (PHTub-1-3) or shifting it to the opposite side of the ring (PHTub-7-9) could permit cytotoxicity. This was indeed confirmed. In these series, the position of the ethyl group was the major determinant of both isomers' bioactivity, which largely corresponded between the sets (e.g. PHTub-1 corresponded to PHTub-7). PHTub-2/8's bioactivity was photoswitchable, with the $Z$-isomers ca. twice as potent as the $E$ -

\footnotetext{
${ }^{1 *}$ Majority-(or all-) $Z$ by green illumination at $525-535 \mathrm{~nm}$ or dark conditions, ${ }^{*}$ majority- $E$ by blue illumination at $435-450 \mathrm{~nm}$; $44 \mathrm{~h}$ incubation; illuminations pulsed at $75 \mathrm{~ms}$ per $15 \mathrm{~s}$ with $<1 \mathrm{~mW} / \mathrm{cm}^{2}$;
}

isomers, and they achieved excellent potency that is superior to all photoswitchable tubulin binders so far reported ${ }^{3,21,24}$, and nearly on par with non-photoswitchable colchicine site binders used as drugs (PHTub-8 $\mathrm{IC}_{50} \leq 120 \mathrm{nM}$, colchicine typically ca. $20 \mathrm{nM}$ ). Despite sacrificing some potency, shifting the ethyl group in PHTub-1/ 7 greatly improved the photoswitchability of bioactivity, which is a necessary compromise for effective photopharmaceuticals. E-PHTub-1 had good potency under blue illumination (mostly-E-PHTub-1 IC 50 ca. $2 \mu \mathrm{M}$ in Jurkat and HeLa), while ZPHTub-1 displayed only weak and irreproducible bioactivity above $10 \mu \mathrm{M}$ which we attributed to its moderate solubility. We have previously observed that the out-of-plane middle methoxy group of a 3,4,5-trimethoxyphenyl motif brings significant solubility benefits compared to dimethoxyphenyl. ${ }^{3}$ We were delighted to find this reproduced in PHTub-7, which retained good potency under blue illumination (mostly-E-PHTub-7 $\mathrm{IC}_{50} \mathrm{ca} .3-5 \mu \mathrm{M}$ in Jurkat and $\mathrm{HeLa}$ ) yet gave no visible effects under green illumination or in the dark $(<10 \%$ antiproliferative effect up to $50 \mu \mathrm{M})$ which we attribute to its improved solubility suppressing aggregation effects (Fig 3a-b). Further shifting the ethyl group showed that PHTub1/7 indeed represent a local optimum for photoswitchability of bioactivity: PHTub-3 was active $\left(E-\mathrm{IC}_{50}=\right.$ $3 \mu \mathrm{M}$ ) but only showed mediocre (i.e. 3-fold) photoswitchability of bioactivity, while PHTub-9 was similarly but not strongly active in each isomer. PHTub-NMe in which the N-methyl group similarly acts a spacefilling group adjacent to the double bond, was inactive as both isomers (Table 1).

Table 1. Tabulated $\mathrm{IC}_{50}$ values of all PHTubs in HeLa or Jurkat cell lines as ${ }^{*}$ majority- $Z$ and ${ }^{*}$ majority- $E$ isomer mixtures. ${ }^{1}$

\begin{tabular}{|c|cc|cc|}
\hline \multirow{2}{*}{ compound } & \multicolumn{2}{|c|}{ IC $_{50}$ HeLa $[\mu \mathrm{M}]$} & \multicolumn{2}{c|}{ IC $_{50}$ Jurkat $[\mu \mathrm{M}]$} \\
\cline { 2 - 5 } & Z-PHTub* & E-PHTub* & Z-PHTub* $^{*}$ & E-PHTub* \\
\hline PHTub-1 & $>30$ (n.sol.) & 1.8 & 10 & 2 \\
PHTub-2 & 0.2 & 0.4 & 0.1 & 0.3 \\
PHTub-3 & 13 & 3.2 & 9 & 3.5 \\
PHTub-4 & $>100$ & ca. 9 & 4 & ca. 20 \\
PHTub-5 & $>100$ & 11 & $>100$ & 3 \\
PHTub-6 & $>20$ (n.sol.) & ca. 20 (n.sol.) & $>20$ (n.sol.) & $>20$ (n.sol.) \\
PHTub-7 & $>50$ (n.sol.) & 4.5 & $>50$ (n.sol.) & 3.5 \\
PHTub-8 & 0.07 & 0.12 & 0.03 & 0.05 \\
PHTub-9 & 6.3 & 5.5 & 4.5 & 4.3 \\
PHTub-NMe & $>20$ (n.sol.) & ca. 20 (n.sol.) & $>20$ (n.sol.) & ca. 20 (n.sol.) \\
\hline
\end{tabular}

Taken together, the ordered structure-activity relationships of the PHTubs (first trimethoxy orientation, then spacefill orientation) can be understood with reference to those expected for colchicine site binders. The demonstration of light-independently-inactive permutation controls PHTub-3/9 as well as highly- but light-independently-active PHTub-2/8 argues that phototoxicity or other nonspecific toxicities are not an inherent problem of the PHT scaffold. Due to its excellent photoswitchability, we proceeded for further biological testing with PHTub-7 as our lead PHT photopharmaceutical. In parallel, we used PHTub-2/8 interchangeably as low-photoswitchability but highly bioactive controls likely to share its mechanism of action. three independent experiments; $n$.sol. indicates apparent bioactivity near the solubility limit which we attribute to nonspecific aggregation effects; see Supporting Information for details. 

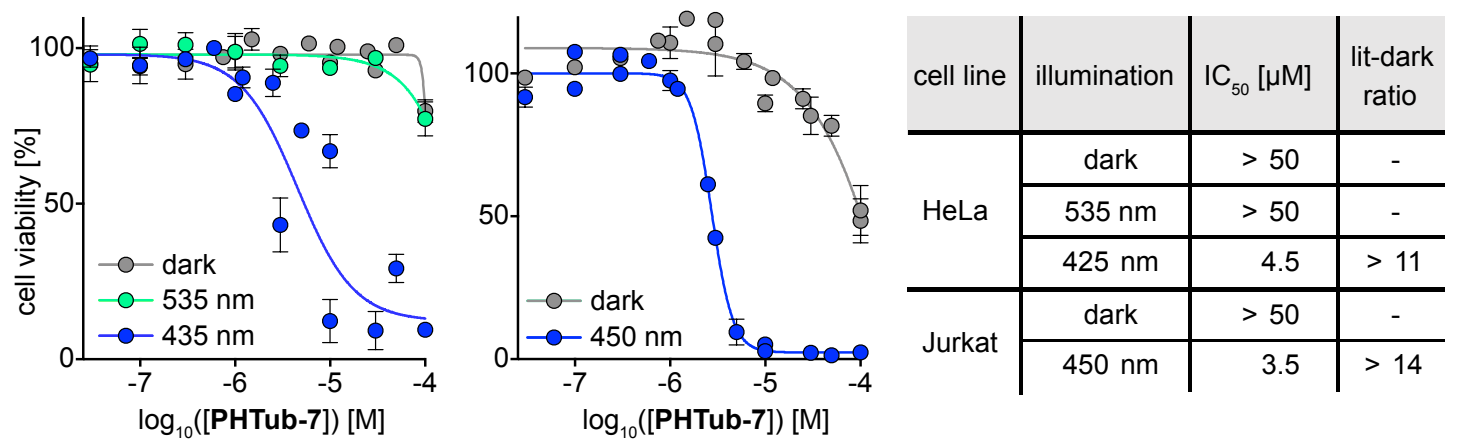

C

PHTub-2

PHTub-7

PHTub-8
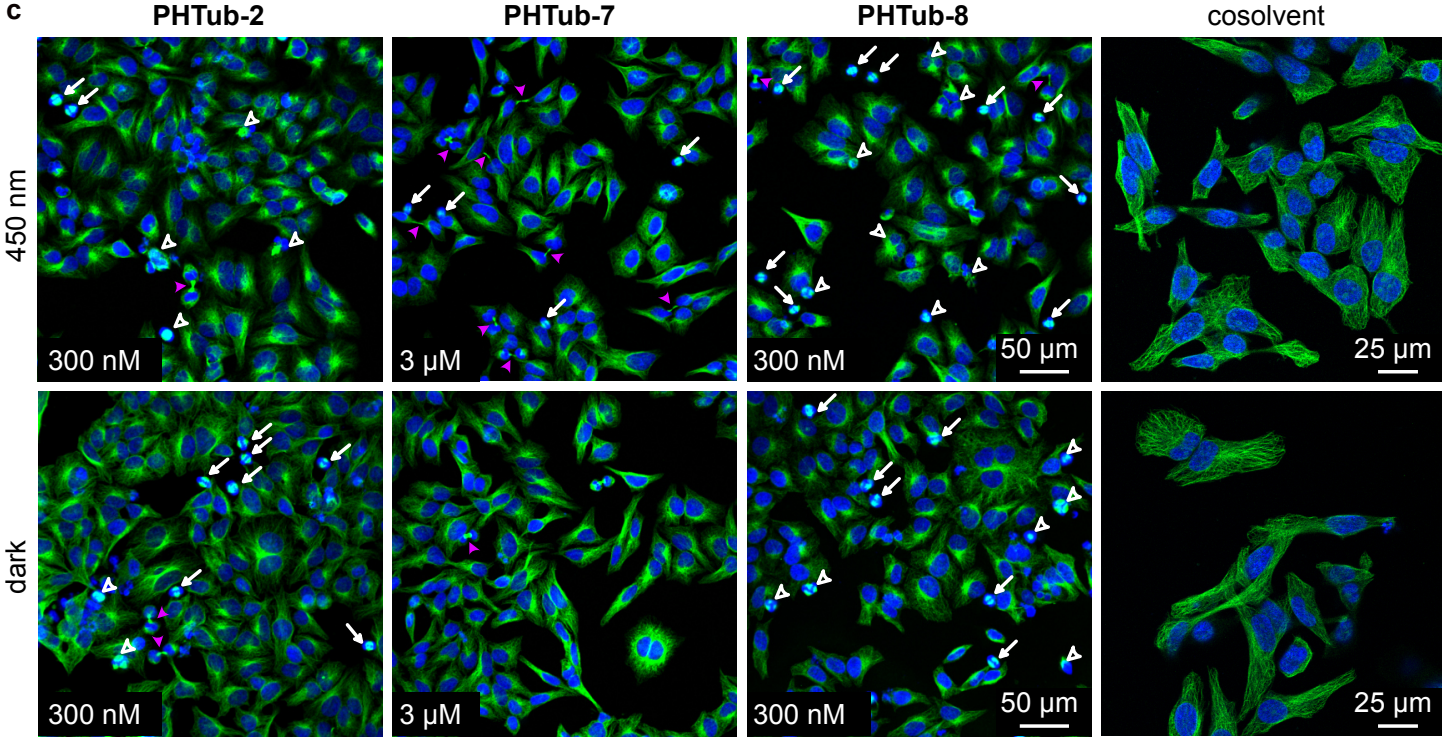

Figure 3 - PHTub bioactivity is light-specific and tubulin-mediated. (a-b) PHTub-7 gave strong light-specific antiproliferative effects with wavelength-dependency that matched the expectations from cell-free PSS measurements (HeLa and Jurkat cells). (c) Immunofluorescence imaging of HeLa cells treated with PHTub-2/7/8 or cosolvent only, under pulsed $450 \mathrm{~nm}$ illumination or dark conditions. White arrows indicate mitotic arrests, hollow white arrowheads indicate multipolar spindles and other mitotic failures, filled pink arrowheads indicate remnant cytokinetic bridges. (12 h incubation; $\alpha$-tubulin in green, DNA stained with DAPI in blue, scale bars represent $50 \mu \mathrm{m}$ in PHTub-treated cells and $25 \mu \mathrm{m}$ in cosolvent-only control cells).

\section{Tubulin-specific mechanism of action in cells}

To test the tubulin-specificity of their light-dependent cytotoxicity, we first imaged the endogenous microtubule (MT) network architecture in cells incubated for 12 hours with PHTubs. The nanomolar-potency PHTub-2/8 caused a high proportion of mitotic arrests (arrows) and multipolar spindles or other mitotic failures (hollow arrowheads) under both illumination conditions (Fig 3c), reflecting expectations from their cytotoxicity assays. PHTub-7 was less potent in its effects, matching the cytotoxicity results: but the $450 \mathrm{~nm}$-specific accumulation of mitotic arrests and of abnormal remnant cytokinetic bridges (filled arrowheads) a hallmark of perturbed mitosis ${ }^{26}$ - was notable, whereas this was almost not seen in dark conditions (Fig 3c). A slight $E$-specific reduction of microtubule polymer mass was also indicated, again with no structural effect at the same concentrations in the dark. We therefore concluded that PHTubs could represent a valid design for tubulin inhibitors targeting the colchicine binding site, and proceeded to secondary mechanistic assays.

To crosscheck if the major cellular bioactivity mechanism of the PHTubs is MT inhibition, we examined their ability to induce light-dependent $\mathrm{G}_{2} / \mathrm{M}$-phase cell cycle arrest. ${ }^{22}$ Using flow cytometric analysis we observed, as expected, induction of $\mathrm{G}_{2} / \mathrm{M}$ arrest for E-PHTub-7 but not for Z-PHTub-7 (Fig 4), while PHTub-2 gave potent but nearly light-independent cell cycle arrest (Fig S8). This further supports that E-PHTub-7 potently inhibits MT function while Z-PHTub-7 does not, suiting it to photoswitching-based control of MT-dependent processes.

\section{Live cell photocontrol of microtubule dynamics by in situ PHTub photoswitching}

We next tested the suitability of PHTubs (and by extension, the PHT scaffold) for spatially and temporally precise photoswitching uses during live cell confocal microscopy. This is a significant advance for photopharmacology, since as far as we know, no hemithioindigo derivatives have yet been demonstrated in temporally-resolved live cell studies with spatially-resolved in situ photoswitching. To do so, we imaged microtubule dynamics in HeLa cells transfected with fluorescently-labelled end binding proteins (EBs). EBs mark the GTP cap regions of polymerising MTs, so imaging EBs reveals the plus ends of polymerising MTs as dynamic comets. ${ }^{27}$ Imaging EBs during photoswitching is an established spatiotemporally resolved readout for isomerisation-dependent inhibition of MT dynamics by photoswitchable inhibitors. ${ }^{3,28}$ 


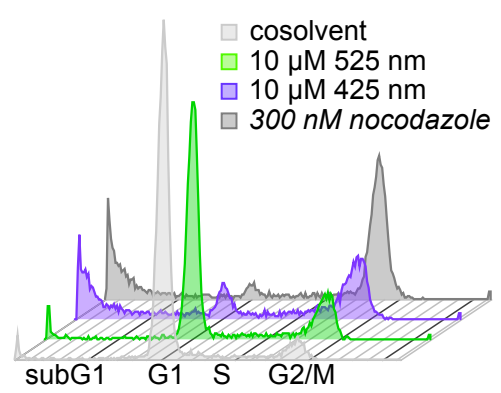

b

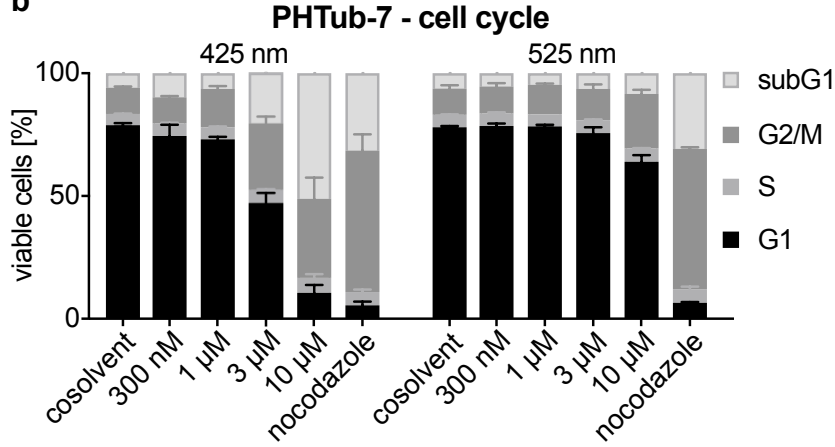

Figure 4 - PHTub can photocontrol cell cycle. (a-b) Cell cycle analysis of cells treated with PHTub-7 under 425 nm pulsing shows significant $\mathrm{G}_{2} / \mathrm{M}$ arrest and cell death (sub-G $\mathrm{G}_{1}$ phase cells), similar to that seen with reference MT inhibitor nocodazole; but PHTub-7 has no effects under $525 \mathrm{~nm}$ pulsing (compare to cosolvent-only DMSO controls).

We first confirmed that Z-PHTub-7 (dark state) has no significant residual effects on MT dynamics, and also avoids photoisomerisation during imaging at $561 \mathrm{~nm}$ (used for imaging $\mathrm{RFP} / \mathrm{mCherry} / \mathrm{tdT}$ omato fusion proteins). We imaged MT dynamics using tdTomato-EB3, showing that while only $561 \mathrm{~nm}$ is applied, Z-PHTub-7 caused no significant change of MT dynamics compared to DMSO controls (Fig 5).

Next, we applied $442 \mathrm{~nm}$ focussed laser pulses to photoisomerise PHTub-7 in situ to the E-isomer throughout the field of view. This drastically suppressed cellular MT dynamics with high temporal precision (Fig 5a-b). MT dynamics did not recover over minutes; in light of further experiments, we believe this indicates that E-PHTub-7 does not rapidly diffuse out of cells because it is lipophilic (intracellular "sinks"29), rather than relating to potential bulk photoisomerisation in the medium (see below).

$442 \mathrm{~nm}$ is nearly an ideal photoactivation wavelength for PHTs (Table S3), but it is not a very common laser line on the confocal
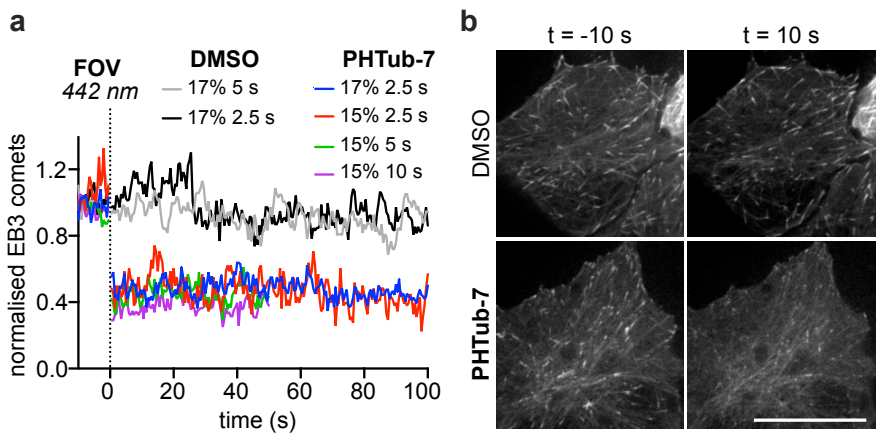

C

e
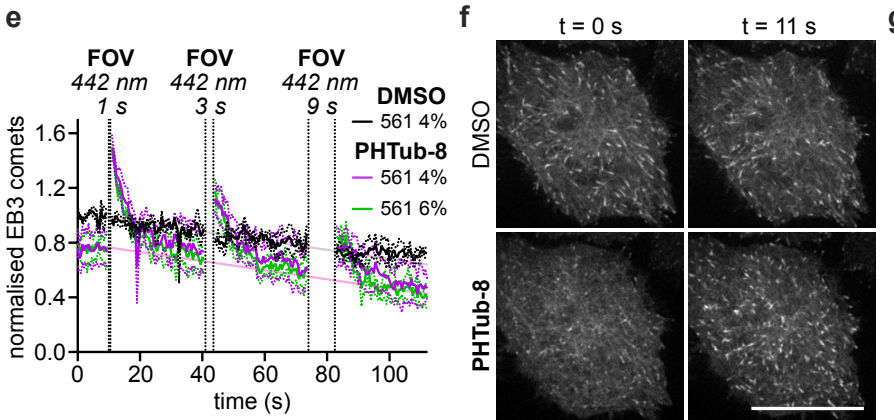
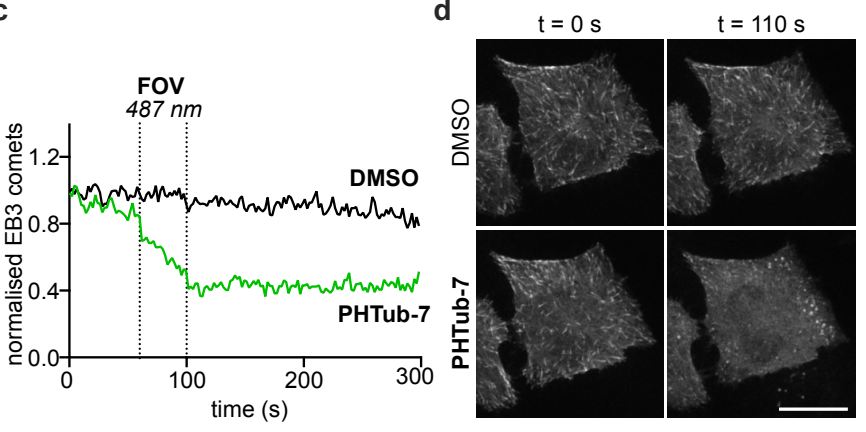

microscopes where MT research is performed. However, GFPexciting lasers (typically 487,488 , or $491 \mathrm{~nm}$ ) are standard on all confocal microscopes. We therefore tested whether GFP lasers are also suitable for cellular photoactivation of PHTub-7. Indeed, $487 \mathrm{~nm}$ illumination also allowed reproducible induction of MT dynamics inhibition (Fig 5c-d; Movie S1), though more slowly than $442 \mathrm{~nm}$ at comparable intensity. These useful results show that PHT-based photopharmaceuticals can be isomerised on any standard confocal microscope using cyan or blue lasers that have little potential for phototoxicity in cell culture research. This can be contrasted favourably to most photocages and indeed most azobenzene-based photopharmaceuticals that require UV $(355 \mathrm{~nm}$ laser) or near-UV ( $405 \mathrm{~nm}$ laser) photoactivations. We also found that cellular MT dynamics were unaffected by similar intensity $405 \mathrm{~nm}$ laser illuminations of PHTub-7 and PHTub-8 (Movie S2) suggesting that quantum yields of isomerisation of the PHTs are more complex in cellular settings than in cell-free conditions (c.f. Fig 2c).
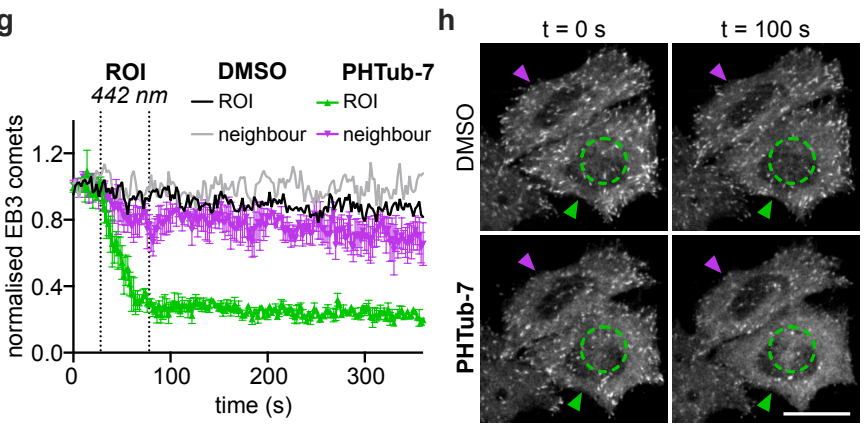

Figure 5 - PHTubs enable in situ photoswitching of MT dynamics in live cells. (a-d) MT dynamics are temporally-precisely suppressed when cells treated with lit-active Z-PHTub-7 $(2 \mu \mathrm{M})$ are illuminated within the field of view (FOV) at (a-b) $442 \mathrm{~nm}$ or at (c-d) $487 \mathrm{~nm}$ (data related to Movie S1). (e-f) Cells treated with dark-active Z-PHTub-8 $(0.5 \mu \mathrm{M})$ have suppressed MT dynamics, which can be repeatedly accelerated by illuminations at $442 \mathrm{~nm}$ (data related to Movie S3). Rough photobleaching background rate fits (grey: DMSO, pink: treated) are guides to the eye to distinguish 
accelerations. (g-h) Cell-precise MT dynamics suppression is caused by $442 \mathrm{~nm}$ photoactivation of Z-PHTub-7 ( $2 \mu \mathrm{M})$ within a subcellular region of interest (shown by green circle) in a target "ROI" cell (green arrowhead), while adjacent "neighbour" cells (purple arrowhead) also bathed in Z-PHTub7 but without photoactivation, do not show greatly altered MT dynamics (data related to Movie S4). (EB3-tdTomato-transfected HeLa cells; tdTomato imaged at $561 \mathrm{~nm}$; EB3 "comet" counts quantify dynamic MTs; comet counts normalised to the average of the first 5 frames of treated or DMSO cells as appropriate; $\mathrm{a} / \mathrm{c}: \mathrm{n}=1$ cell per condition, $\mathrm{e} / \mathrm{g}$ : mean \pm SEM comet counts, $\mathrm{n}=3$ cells per condition; $\mathrm{c} / \mathrm{e} / \mathrm{g}$ : dotted vertical lines indicate start and finish of $487 \mathrm{~nm}$ or $442 \mathrm{~nm}$ phases; b/d/f/h: scale bars indicate $20 \mu \mathrm{m}$; for full caption and details see Supporting Information).

To crosscheck the mechanism of action of PHTub-7, we also imaged its potent congener PHTub-8 which had appeared more active as its dark $(Z)$ isomer than in its lit state. Encouragingly, we observed both that Z-PHTub-8 suppresses MT dynamics, and that in situ 442 or $487 \mathrm{~nm}$ illumination caused drastic acceleration of MT polymerisation for a short while. Photoaccelerations could typically be performed at least three times before their magnitude became less impressive (Fig 5e-f; Movie S3). These results match the cytotoxicity and immunofluorescence assays, supporting that both PHTub7/8 directly and photoswitchably inhibit tubulin. Though at first glance it might seem paradoxical that PHTub-8 was temporally reversible while PHTub-7 was not, we believe this has a consistent explanation (see Discussion).

Lastly, we performed cell-precise photoisomerisations of PHTub-7 in single selected cells, hoping to temporally precisely block MT dynamics in those cells, without affecting their neighbour cells. In cells targeted with $442 \mathrm{~nm}$ laser regions (ROIs), EB3 comet counts dropped even below what had been seen with full field of view photoactivations, and did not recover over minutes, while neighbour cells remained unaffected as in non-treated and non-illuminated cosolvent controls (Fig 5g-h; Movie S4). This supports the idea of intracellular "sinks"29 of slowly-diffusing PHTub responsible for persistent effects, by excluding any effects of bulk photoisomerisation in the medium. (It also contrasts to diffusional recovery $(\sim 30 s)$ we have observed for other scaffolds in similar conditions, such as the styrylbenzothiazole Z-SBTub3 ${ }^{24}$ ).

Thus, PHTub-7 can inhibit MT dynamics of target cells with temporal precision on the scale of seconds and spatial precision on the single-cell level. Taken together, these experiments demonstrate that the PHT scaffold may in general be uniquely suited for in situ photoswitching use in live cells, as part of both dark- or litactive photopharmaceuticals against other protein targets.

\section{DISCUSSION}

Photopharmacological approaches to high-spatiotemporal-precision manipulation studies of endogenous proteins have progressed significantly, enabling biological application to membranes, ion channels, and the cytoskeleton. ${ }^{1,6,30}$ Photopharmaceuticals for the cytoskeleton have transitioned particularly rapidly beyond cellular proofs of concept for non-invasive, reversible, cellularly-resolved optical control over MT dynamics and network structure in cell culture. Despite the relatively poor functional dynamic range of the azobenzene-based PSTs (3-fold at $405 / 514 \mathrm{~nm}$ ), they have been applied to powerful studies in embryos of fruit fly Drosophila melanogaster ${ }^{31}$, worm Caenorhabditis elegans $^{3}$, zebrafish Danio rerio ${ }^{32}$, and mouse Mus musculus $s^{33}$, where they have helped to resolve questions in development and in neuroscience ${ }^{34,35}$. Such uses illustrate the power of photopharmacology to enable previously inaccessible studies of spatiotemporally complex processes.

However, the scope of photopharmacology remains restricted by the photochemical and biochemical limitations of its photoswitch scaffold repertoire. Several limitations of the major scaffold azobenzene, including the diazene's susceptibility to thiol- mediated degradation and its restricted scope of substituents compatible with bistable photoswitch performance, are relatively wellknown. ${ }^{24}$ Non-azobenzene photoswitches that avoid these pitfalls, such as dithienylethenes, hemithioindigos, and heterostilbenes have recently begun to be used in bioactive pharmacophores ${ }^{16,24,36}$; and the development of new photoswitch scaffolds is a valuable research goal in general. ${ }^{37}$ Yet, while some recently developed scaffolds have approached near-quantitative bidirectional photoswitching in physiological media, there is still no clear choice of scaffold that can robustly ensure this performance in cells using the light sources that are actually available for biological research.

In this research, we show that PHT may be a general photopharmaceutical alternative to azobenzenes, offering comparable size and chemical simplicity, but better GSH resistance, more cellcompatible photoisomerisation wavelengths, and a better match of its optimum photoisomerisation wavelengths to those available on all confocal microscopes.

We had particularly sought to develop a photopharmaceutical with a high functional dynamic range. Towards this end, we hoped that due to its hydrophobicity favouring cellular biolocalisation in water-excluded environments, the PHTubs' cellular photoswitching could approach the excellent PSSs that PHT shows in organic media, rather than the poor photoswitching reported in protic me$\operatorname{dia}^{19}$. This is not yet generally recognised as a design opportunity for photopharmacology, but we believe it is an important one. Tuning of photochemistry dependent on cellular environment has long been exploited in fluorescence microscopy (e.g. the 20-fold enhancement of DAPI fluorescence upon DNA binding $)^{38}$, and has been observed for photoswitches (e.g. hypsochromic shifts of optimum $E$-to- $Z$ isomerisation wavelength in membranes ${ }^{39}$ ). The strong photoswitchability of bioactivity observed with PHTub-7 in this work suggests that the favourable organic-media photochemistry of this heteroaryl hemithioindigo can indeed be harnessed for cellular applications, and opens a more nuanced perspective for the design of photopharmaceuticals that can bring high functional dynamic range photoswitching to biological investigations.

Additionally, through PHTub-8, we have given the first demonstration of time-resolved live cell photocontrol of a dark-active cytoskeleton photopharmaceutical. Though its reversibility was limited, this achievement is conceptually significant, because reversible dark-active photocontrol over biological processes using stoichiometric inhibitors is conceptually more challenging to achieve than with highly nonlinearly-acting modulators, as have been used to control e.g. excitable cell firing ${ }^{40}$. Stoichiometric control requires (i) high in situ photoconversion to the less bioactive metastable isomer (i.e. high practical FDR), which is typically problematic for photoswitch scaffolds (other than PHTs), as well as (ii) high isomer-specificity of bioactivity, which is typically problematic for their bioactive derivatives (other than some colchicinoids). Although E-PHTub-8 clearly had significant residual bioactivity, its high FDR - achieved by matching the PHT scaffold's peak isomerisation efficiency to the GFP laser - was crucial for success in photocontrol. We also believe that enhanced dark-active compounds 
based on PHTub-8 will be useful tools for localised induction of MT polymerisation in biology, which remains a high-value goal of reagent development ${ }^{28,41}$.

It was interesting that PHTub-8 appeared somewhat reversible while PHTub-7 did not (Fig 5e,g). We propose that this arises since upon $442 / 487 \mathrm{~nm}$ photoisomerisation, partial E-PHTub-8 unbinding from tubulin monomers "injects" tubulin into the polymerisable pool, so polymerisation rate and comet count increase. This excess is then 'used up' by addition to MT polymer; and over ca. $10 \mathrm{~s}$ the cell returns to steady state dynamics. Thus at each subsequent $442 / 487 \mathrm{~nm}$ photoisomerisation pulse, there is somewhat less tubulin monomer that can be injected, and rate enhancements become less pronounced. We stated that typically three cycles could be performed where accelerations are obvious; but note that this limit is not only biological - it partially arises since accelerations must be distinguished against a continuous background of comet decrease from marker photobleaching during imaging (pink fit in Fig 5e) as has also previously been described, ${ }^{3}$ and this bleaching restricts the potential for imaging the system. We have discounted three alternative considerations for reversibility which are usually significant for photopharmacology: (1) due to the results of e.g. Fig $\mathbf{5 g}$ we proposed that PHTubs do not rapidly diffuse out of cells; under equilibrated conditions we assume that diffusion into cells is also relatively slow; therefore we do not assign the fast reversibility only of PHTub-8 to diffusion. (2) We believe that spontaneous relaxation is too slow to be involved $\left(\mathrm{t}_{1 / 2}\right.$ probably >> $15 \mathrm{~min}$ in primarily water-excluded environments), and see no reasons that it would be so rapid for PHTub8 while being insignificant for PHTub7. (3) We excluded photoreversion of PHTub8 by experiments where we increased imaging laser power but saw no rate change (Fig 5e; Movie S3). These considerations can have general interest for photopharmacology.

\section{CONCLUSIONS}

In this work we have applied the pyrrole hemithioindigo (PHT) photoswitch scaffold to develop a rationally-designed series of photopharmaceutical reagents for photocontrol of the microtubule (MT) cytoskeleton: a critical biological target in urgent need of better reagents for high-resolution modulation assays. This choice of the PHT scaffold was explicitly motivated by its superior photoswitching performance at microscopy laser lines. We have demonstrated these "PHTub" reagents' capacity to robustly apply optical control over MT network integrity, cell division, and cell death in long-term assays. In short-term assays, they achieve temporally-resolved, cell-specific, optical modulation of MT dynamics in live cells. To our knowledge this is the first high-resolution live cell application of any hemithioindigo as a photopharmaceutical pharmacophore; and its success highlights both the PHTubs' and the PHT scaffold's promise for a range of high-spatiotemporal-precision biological studies. We therefore believe that PHTub-7 in particular will be a valuable photopharmaceutical for high-precision cytoskeleton research, with promising applications in the fields of cellular transport, mechanostasis, migration, cell division, and embryonic development.

This research also suggests that the PHT scaffold too will find a broad range of applications to biological targets that are poorly addressed by current photopharmaceuticals. These targets may be located both cytosolically, as well as in water-excluded environments where the PHT scaffold may be even more advantageously applicable. In general, novel photoswitches that can be used to access new photochemical performance without requiring conceptual redesign to retain on-target bioactivity (such as spectrally shifted diazocines, ${ }^{42,43}$ tetra-ortho-substituted azobenzenes ${ }^{12,44}$ and azoniums $\mathrm{s}^{45}$ ) are recognised as high-value targets in photopharmacology. Therefore it is significant that a similar molecular design of PHTub-7 compared to its azobenzene analogues (PSTs) has ensured similar isomer-dependent bioactivity. Our work thus suggests that Zweig and Newhouse's pyrrole hemithioindigos ${ }^{19}$ may be generally useful as a straightforward alternative to azobenzenes in photopharmacology, for powerful photopharmaceuticals with higher functional dynamic ranges under standard microscopy lasers, useful against a range of biological targets.

\section{ASSOCIATED CONTENT}

\section{Supporting Information}

The Supporting Information is available free of charge on the ACS Publications website.

(1) Supporting Information PDF containing (i) chemical synthesis and NMR spectra (ii) photocharacterisation in vitro (iii) biochemistry (iv) biological methods and data.

(2) Movies S1-S4: movies are annotated with blue dots to indicate illumination pulses $(405 / 442 / 487 \mathrm{~nm})$, timestamps in mm:ss, and $20 \mu \mathrm{m}$ scalebars; all show HeLa cells transfected with EB3-tdTomato, imaged at $561 \mathrm{~nm}$ in 1\% DMSO, with/without PHTub as indicated; captions are as follows:

Movie S1, temporally precise inhibition of cellular MT polymerisation dynamics by photoactivation of lit-active Z-PHTub-7 at $487 \mathrm{~nm}$, related to Figure 5c-d: cells bathed in 1\% DMSO show no inhibition of MT polymerisation dynamics unless both lit-active $Z$ PHTub-7 $(2 \mu \mathrm{M})$ and then a $487 \mathrm{~nm}$ illumination pulse are applied. Movie S2, Z-PHTub-7/8 are not effectively photoactivated at $405 \mathrm{~nm}$, related to Figure 5c-f: no change of MT polymerisation dynamics is elicited by $405 \mathrm{~nm}$ illumination of lit-active Z-PHTub-7 ( $2 \mu \mathrm{M}$; no inhibition induced) or dark-active Z-PHTub-8 (0.5 $\mu \mathrm{M}$; no reduction of inhibition).

Movie S3, temporally precise reduction of inhibition of cellular MT polymerisation dynamics by photoactivation of dark-active Z-PHTub-8 at 487 or 442 nm, related to Figure 5e-f: cells bathed in dark-active Z-PHTub-8 $(0.5 \mu \mathrm{M})$ have inhibited MT polymerisation dynamics until 487 or $442 \mathrm{~nm}$ illumination pulses are applied; the DMSO-only control is unaffected by pulsing.

Movie S4, Cell-localised ROI illumination of cells globally treated with lit-active PHTub-7 induces cell-precise, temporally resolved MT polymerisation inhibition in the ROI-target cell only, related to Figure 5g-h: cells bathed in lit-active Z-PHTub-7 $(2 \mu \mathrm{M})$ have uninhibited MT polymerisation dynamics. A single cell was targeted with an ROI illumination pulse at $442 \mathrm{~nm}$, which blocks MT dynamics in that cell without affecting neighbour cells. The DMSO-only control (prior to PHTub application) shows no change of MT dynamics during the imaging and illumination protocol.

\section{AUTHOR INFORMATION}

\section{Corresponding Author}

oliver.thorn-seshold@cup.lmu.de

\section{Author Contributions}

A.S. designed targets and performed synthesis, photocharacterisation, and in vitro studies. J.C.M.M. performed live cell imaging studies. C.H. performed long-term cellular studies. L.N.P assisted A.S. in synthesis. A.A. supervised live cell studies. J.T.-S. performed and supervised longterm cellular studies and coordinated data assembly. O.T.-S. 
designed the study, supervised all other experiments, coordinated data assembly and wrote the manuscript.

\section{Funding Sources}

J.T.-S. thanks the Joachim Herz foundation for grant support. O.T.-S. thanks the German Research Foundation (DFG: SFB 1032 project B09 number 201269156, Emmy Noether grant number 400324123, SFB TRR 152 project P24 number 239283807, SPP 1926 project XVIII number 426018126) and the Munich Centre for NanoScience initiative (CeNS) for funding. J.C.M.M. acknowledges support from an EMBO Long Term Fellowship

\section{ACKNOWLEDGEMENT}

We thank Y. Kraus and D. Lonken (LMU) for early PHTub viability testing and Marie Delattre (ENS Lyon, FR) and Wallis Nahaboo (University of Brussels, BE) for early proofing of cellular photoswitchability of hemithioindigos.

\section{REFERENCES}

(1) Rullo, A.; Reiner, A.; Reiter, A.; Trauner, D.; Isacoff, E. Y.; Woolley, G. A. Long Wavelength Optical Control of Glutamate Receptor Ion Channels Using a Tetra-Ortho-Substituted Azobenzene Derivative. Chem. Commun. 2014, 50 (93), 14613-14615. https://doi.org/10.1039/C4CC06612J.

(2) Agnetta, L.; Kauk, M.; Canizal, M. C. A.; Messerer, R.; Holzgrabe, U.; Hoffmann, C.; Decker, M. A Photoswitchable Dualsteric Ligand Controlling Receptor Efficacy. Angew. Chem. Int. Ed. 2017, 56 (25), 7282-7287. https://doi.org/10.1002/anie.201701524.

(3) Borowiak, M.; Nahaboo, W.; Reynders, M.; Nekolla, K.; Jalinot, P.; Hasserodt, J.; Rehberg, M.; Delattre, M.; Zahler, S.; Vollmar, A.; Trauner, D.; Thorn-Seshold, O. Photoswitchable Inhibitors of Microtubule Dynamics Optically Control Mitosis and Cell Death. Cell 2015, 162 (2), 403-411. https:// doi.org/10.1016/j.cell.2015.06.049.

(4) Klán, P.; Šolomek, T.; Bochet, C. G.; Blanc, A.; Givens, R.; Rubina, M.; Popik, V.; Kostikov, A.; Wirz, J. Photoremovable Protecting Groups in Chemistry and Biology: Reaction Mechanisms and Efficacy. Chem. Rev. 2013, 113 (1), 119-191. https://doi.org/10.1021/cr300177k.

(5) Hüll, K.; Morstein, J.; Trauner, D. In Vivo Photopharmacology. Chemical Reviews 2018, 118 (21), 10710-10747. https://doi.org/10.1021/acs.chemrev.8b00037.

(6) Borowiak, M.; Küllmer, F.; Gegenfurtner, F.; Peil, S.; Nasufovic, V.; Zahler, S.; Thorn-Seshold, O.; Trauner, D.; Arndt, H.-D. Optical Manipulation of F-Actin with Photoswitchable Small Molecules. J. Am. Chem. Soc. 2020, 142 (20), 9240-9249. https://doi.org/10.1021/jacs.9b12898.

(7) Castle, B. T.; Odde, D. J. Optical Control of Microtubule Dynamics in Time and Space. Cell 2015, 162 (2), 243-245. https://doi.org/10.1016/j.cell.2015.06.064.

(8) Wittmann, T.; Dema, A.; van Haren, J. Lights, Cytoskeleton, Action: Optogenetic Control of Cell Dynamics. Current Opinion in Cell Biology 2020, 66, 1-10. https://doi.org/10.1016/j.ceb.2020.03.003.

(9) Florian, S.; Mitchison, T. J. Anti-Microtubule Drugs. In The Mitotic Spindle: Methods and Protocols; Chang, P., Ohi, R., Eds.; Springer New York: New York, NY, 2016; pp 403-421.

(10) Peterson, J. R.; Mitchison, T. J. Small Molecules, Big Impact: A History of Chemical Inhibitors and the Cytoskeleton. Chem. Biol. 2002, 9 (12), 1275-1285. https://doi.org/10.1016/S1074-5521(02)00284-3.

(11) Fuchter, M. J. On the Promise of Photopharmacology Using Photoswitches: A Medicinal Chemist's Perspective. J. Med. Chem. 2020 https://doi.org/10.1021/acs.jmedchem.0c00629.

(12) Konrad, D. B.; Savasci, G.; Allmendinger, L.; Trauner, D.; Ochsenfeld, C.; Ali, A. M. Computational Design and Synthesis of a Deeply Red-Shifted and Bistable Azobenzene. J. Am. Chem. Soc. 2020. https://doi.org/10.1021/jacs.9b10430.

(13) Weston, C. E.; Richardson, R. D.; Haycock, P. R.; White, A. J. P.; Fuchter, M. J. Arylazopyrazoles: Azoheteroarene Photoswitches
Offering Quantitative Isomerization and Long Thermal Half-Lives. J. Am. Chem. Soc. 2014, 136 (34), 11878-11881. https://doi.org/10.1021/ja505444d.

(14) Gaspari, R.; Prota, A. E.; Bargsten, K.; Cavalli, A.; Steinmetz, M. O. Structural Basis of Cis- and Trans-Combretastatin Binding to Tubulin. Chem 2017, 2 (1), 102-113. https://doi.org/10.1016/j.chempr.2016.12.005.

(15) Gao, L.; Meiring, J. C. M.; Wranik, M.; Heise, C.; Distel, M.; Bausch, A.; Llobet, A.; Cabernard, C.; Steinmetz, M. O.; Thorn-Seshold, J.; Akhmanova, A.; Thorn-Seshold, O. In Vivo Microtubule Photocontrol with High-Potency, GFP-Orthogonal Photoswitchable Inhibitors. bioRxiv (in submission) 2021.

(16) Sailer, A.; Ermer, F.; Kraus, Y.; Lutter, F.; Donau, C.; Bremerich, M.; Ahlfeld, J.; Thorn-Seshold, O. Hemithioin digos as Desymmetrised Molecular Switch Scaffolds: Design Control over the Isomer-Dependency of Potent Photoswitchable Antimitotic Bioactivity in Cellulo. ChemBioChem 2019, 20, 1305-1314. https://doi.org/10.1002/cbic.201800752.

(17) Gavin, J.; Ruiz, J. F. M.; Kedziora, K.; Windle, H.; Kelleher, D. P.; Gilmer, J. F. Structure Requirements for Anaerobe Processing of Azo Compounds: Implications for Prodrug Design. Bioorg. Med. Chem. Lett. 2012, 22 (24), 7647-7652. https://doi.org/10.1016/j.bmcl.2012.10.014.

(18) Nikolaev, D. M.; Panov, M. S.; Shtyrov, A. A.; Boitsov, V. M.; Vyazmin, S. Yu.; Chakchir, O. B.; Yakovlev, I. P.; Ryazantsev, M. N. Perspective Tools for Optogenetics and Photopharmacology: From Design to Implementation. In Progress in Photon Science: Recent Advances; Yamanouchi, K., Tunik, S., Makarov, V., Eds.; Springer International Publishing: Cham, 2019; pp 139-172. https://doi.org/10.1007/978-3-030-05974 38

(19) Zweig, J.E.; Newhouse, T. R. Isomer-Specific Hydrogen Bonding as a Design Principle for Bidirectionally Quantitative and Redshifted Hemithioindigo Photoswitches. J. Am. Chem. Soc. 2017, 139 (32), 1095610959. https://doi.org/10.1021/jacs.7b04448.

(20) Zweig, J. E.; Ko, T. A.; Huang, J.; Newhouse, T. R. Effects of $\pi$ Extension on Pyrrole Hemithioindigo Photoswitches. Tetrahedron 2019, 75 (34), 130466. https://doi.org/10.1016/j.tet.2019.130466.

(21) Sailer, A.; Ermer, F.; Kraus, Y.; Bingham, R.; Lutter, F. H.; Ahlfeld, J.; Thorn-Seshold, O. Potent Hemithioindigo-Based Antimitotics Photocontrol the Microtubule Cytoskeleton in Cellulo. Beilstein Journal of Organic Chemistry 2020, 16, 125-134. https://doi.org/10.3762/bjoc.16.14.

(22) Tron, G. C.; Pirali, T.; Sorba, G.; Pagliai, F.; Busacca, S.; Genazzani, A. A. Medicinal Chemistry of Combretastatin A4: Present and Future Directions. J. Med. Chem. 2006, 49 (11), 3033-3044. https://doi.org/10.1021/jm0512903.

(23) Baell, J. B.; Nissink, J. W. M. Seven Year Itch: Pan-Assay Interference Compounds (PAINS) in 2017-Utility and Limitations. ACS Chem. Biol. 2018, 13 (1), 36-44. https://doi.org/10.1021/acschembio.7b00903.

(24) Gao, L.; Meiring, J. C. M.; Kraus, Y.; Wranik, M.; Weinert, T.; Pritzl, S. D.; Bingham, R.; Ntouliou, E.; Jansen, K. I.; Olieric, N.; Standfuss, J.; Kapitein, L. C.; Lohmüller, T.; Ahlfeld, J.; Akhmanova, A.; Steinmetz, M. O.; Thorn-Seshold, O. A Robust, GFP-Orthogonal Photoswitchable Inhibitor Scaffold Extends Optical Control over the Microtubule Cytoskeleton. Cell Chemical Biology 2021, 28, 1-14. https://doi.org/10.1016/j.chembiol.2020.11.007.

(25) Jordan, M. A.; Wendell, K.; Gardiner, S.; Derry, W. B.; Copp, H.; Wilson, L. Mitotic Block Induced in HeLa Cells by Low Concentrations of Paclitaxel (Taxol) Results in Abnormal Mitotic Exit and Apoptotic Cell Death. Cancer Research 1996, 56 (4), 816-825.

(26) Normand, G.; King, R. W. Understanding Cytokinesis Failure. In Polyploidization and Cancer; Poon, R. Y. C., Ed.; Springer New York: New York, NY, 2010; pp 27-55. https://doi.org/10.1007/978-1-44196199-0 3.

(27) Roostalu, J.; Thomas, C.; Cade, N. I.; Kunzelmann, S.; Taylor, I. A.; Surrey, T. The Speed of GTP Hydrolysis Determines GTP Cap Size and Controls Microtubule Stability. Elife 2020, 9 https://doi.org/10.7554/eLife.51992. 
(28) Müller-Deku, A.; Meiring, J. C. M.; Loy, K.; Kraus, Y.; Heise, C.; Bingham, R.; Jansen, K. I.; Qu, X.; Bartolini, F.; Kapitein, L. C.; Akhmanova, A.; Ahlfeld, J.; Trauner, D.; Thorn-Seshold, O. Photoswitchable Paclitaxel-Based Microtubule Stabilisers Allow Optical Control over the Microtubule Cytoskeleton. Nature Communications 2020, 11 (1), 4640. https://doi.org/10.1038/s41467-020-18389-6.

(29) Kathrin M. Scherer; Roger H. Bisby; Stanley W. Botchway; John A. Hadfield; Anthony W. Parker. Anticancer Phototherapy Using Activation of E-Combretastatins by Two-Photon-Induced Isomerization. J. Biomed. Opt. 2014, $20 \quad$ (5), 051004. https://doi.org/10.1117/1.JBO.20.5.051004.

(30) Pritzl, S. D.; Urban, P.; Prasselsperger, A.; Konrad, D. B.; Frank, J. A.; Trauner, D.; Lohmüller, T. Photolipid Bilayer Permeability Is Controlled by Transient Pore Formation. Langmuir 2020. https://doi.org/10.1021/acs.langmuir.0c02229.

(31) Singh, A.; Saha, T.; Begemann, I.; Ricker, A.; Nüsse, H.; Thorn-Seshold, O.; Klingauf, J.; Galic, M.; Matis, M. Polarized Microtubule Dynamics Directs Cell Mechanics and Coordinates Forces during Epithelial Morphogenesis. Nat. Cell Biol. 2018, 20 (10), 1126-1133. https://doi.org/10.1038/s41556-018-0193-1.

(32) Vandestadt, C.; Vanwalleghem, G. C.; Castillo, H. A.; Li, M.; Schulze, K.; Khabooshan, M.; Don, E.; Anko, M.-L.; Scott, E. K.; Kaslin, J. Early Migration of Precursor Neurons Initiates Cellular and Functional Regeneration after Spinal Cord Injury in Zebrafish. bioRxiv 2019, 539940. https://doi.org/10.1101/539940.

(33) Zenker, J.; White, M. D.; Templin, R. M.; Parton, R. G.; ThornSeshold, O.; Bissiere, S.; Plachta, N. A Microtubule-Organizing Center Directing Intracellular Transport in the Early Mouse Embryo. Science 2017, 357 (6354), 925-928. https://doi.org/10.1126/science.aam9335.

(34) Zenker, J.; White, M. D.; Gasnier, M.; Alvarez, Y. D.; Lim, H. Y. G.; Bissiere, S.; Biro, M.; Plachta, N. Expanding Actin Rings Zipper the Mouse Embryo for Blastocyst Formation. Cell 2018, 173 (3), 776-791. https://doi.org/10.1016/j.cell.2018.02.035.

(35) Eguchi, K.; Taoufiq, Z.; Thorn-Seshold, O.; Trauner, D.; Hasegawa, M.; Takahashi, T. Wild-Type Monomeric $\alpha$-Synuclein Can Impair Vesicle Endocytosis and Synaptic Fidelity via Tubulin Polymerization at the Calyx of Held. J. Neurosci. 2017, 37 (25), 6043-6052. https://doi.org/10.1523/jneurosci.0179-17.2017.

(36) Simeth, N. A.; Kneuttinger, A. C.; Sterner, R; König, B. Photochromic Coenzyme Q Derivatives: Switching Redox Potentials with
Light. Chem. Sci. 2017, 8 (9), 6474-6483. https://doi.org/10.1039/c7sc00781g.

(37) Hoorens, M. W. H.; Medved', M.; Laurent, A. D.; Di Donato, M.; Fanetti, S.; Slappendel, L.; Hilbers, M.; Feringa, B. L.; Jan Buma, W.; Szymanski, W. Iminothioindoxyl as a Molecular Photoswitch with 100 $\mathrm{Nm}$ Band Separation in the Visible Range. Nat. Comm. 2019, 10 (1), 2390 https://doi.org/10.1038/s41467-019-10251-8.

(38) Kapuscinski, J. DAPI: A DNA-Specific Fluorescent Probe. null 1995, 70 (5), 220-233. https://doi.org/10.3109/10520299509108199.

(39) Urban, P.; Pritzl, S. D.; Konrad, D. B.; Frank, J. A.; Pernpeintner, C.; Roeske, C. R.; Trauner, D.; Lohmüller, T. Light-Controlled Lipid Interaction and Membrane Organization in Photolipid Bilayer Vesicles. Langmuir 2018, $34 \quad$ (44), 13368-13374. https://doi.org/10.1021/acs.langmuir.8b03241.

(40) Stawski, P.; Sumser, M.; Trauner, D. A Photochromic Agonist of AMPA Receptors. Angewandte Chemie International Edition 2012, 51 (23), 5748-5751. https://doi.org/10.1002/anie.201109265.

(41) Buck, K. B.; Zheng, J. Q. Growth Cone Turning Induced by Direct Local Modification of Microtubule Dynamics. The Journal of Neuroscience 2002, 22 (21), 9358-9367. https://doi.org/10.1523/jneurosci.22-21-09358.2002.

(42) Maier, M. S.; Hüll, K.; Reynders, M.; Matsuura, B. S.; Leippe, P.; Ko, T.; Schäffer, L.; Trauner, D. Oxidative Approach Enables Efficient Access to Cyclic Azobenzenes. J. Am. Chem. Soc. 2019, 141 (43), 1729517304. https://doi.org/10.1021/jacs.9b08794.

(43) Siewertsen, R.; Neumann, H.; Buchheim-Stehn, B.; Herges, R.; Näther, C.; Renth, F.; Temps, F. Highly Efficient Reversible Z-E Photoisomerization of a Bridged Azobenzene with Visible Light through Resolved S1 $\left(\mathrm{N} \pi^{*}\right)$ Absorption Bands. J. Am. Chem. Soc. 2009, 131 (43), 15594-15595. https://doi.org/10.1021/ja906547d.

(44) Bléger, D.; Schwarz, J.; Brouwer, A. M.; Hecht, S. O-Fluoroazobenzenes as Readily Synthesized Photoswitches Offering Nearly Quantitative Two-Way Isomerization with Visible Light. J. Am. Chem. Soc 2012, 134 (51), 20597-20600. https://doi.org/10.1021/ja310323y.

(45) Samanta, S.; Babalhavaeji, A.; Dong, M.; Woolley, G. A. Photoswitching of Ortho-Substituted Azonium Ions by Red Light in Whole Blood. Angew. Chem., Int. Ed. 2013, 52 (52), 14127-14130. https://doi.org/10.1002/anie.201306352. 
Table of Contents artwork

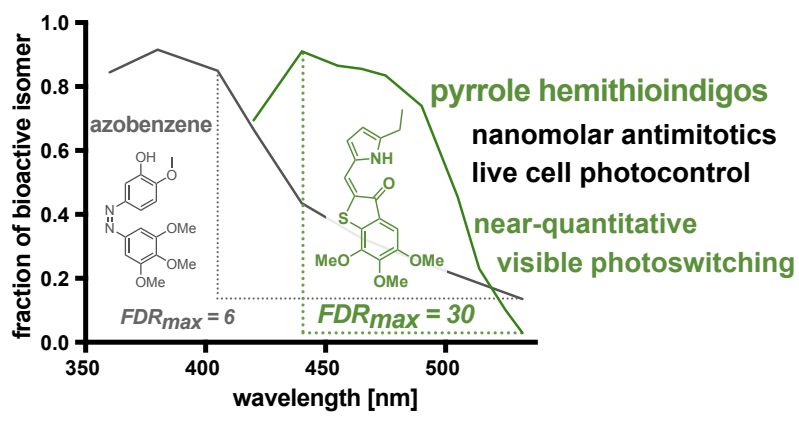

